\title{
Interleukin-8 promotes cell migration via CXCR1 and CXCR2 in liver cancer
}

\author{
HUIJUAN BI, YU ZHANG ${ }^{*}$, SHANSHAN WANG, WENHAO FANG, WENJUN HE, LINA YIN, \\ YING XUE, ZHIXIANG CHENG, MINGHUI YANG and JILU SHEN* \\ Department of Clinical Laboratory, The Fourth Affiliated Hospital of Anhui Medical University, \\ Hefei, Anhui 230000, P.R. China
}

Received October 28, 2018; Accepted July 3, 2019

DOI: $10.3892 / 01.2019 .10735$

\begin{abstract}
Liver cancer (LC), which is one of the most common types of cancer worldwide, is notorious for its high morbidity and mortality rates. Interleukin-8 (IL-8), an important member of the CXC chemokine family that was originally classified as a potent neutrophil chemoattractant, has been shown to serve an important role in inflammation, tumor growth, invasion and metastasis through interactions with its receptors. However, the expression and functional roles of IL-8 and its receptors, CXC chemokine receptor (CXCR) 1 and CXCR2 in the progression of liver cancer remain to be fully elucidated. In the present study, it was shown that the mRNA levels of IL-8, CXCR1 and CXCR2 were increased in peripheral blood mononuclear cells from patients with liver cancer compared with those from patients with cirrhosis or normal controls $(\mathrm{P}<0.05)$. Higher levels of CXCR1, CXCR2 and IL-8 were associated with advanced tumor stage and increased risk of lymph node or distant metastasis. Immunohistochemistry showed that the IL-8, CXCR1 and CXCR2 proteins were expressed in the cytoplasm of hepatoma cells at higher intensities than those of normal controls $(\mathrm{P}<0.05)$. The semi-quantitative analysis revealed that the relative mean density of hepatic IL-8, CXCR1 and CXCR2 staining in liver cancer was significantly increased compared with that in normal liver tissues $(\mathrm{P}<0.05)$. The analysis revealed that the mRNA expression of IL-8 was positively associated with that of CXCR1 $(r=0.618 ; \mathrm{P}<0.05)$ and CXCR2 $(\mathrm{r}=0.569 ; \mathrm{P}<0.05)$. The mRNA levels of CXCR1 and CXCR2 gradually increased with elevated expression of IL-8 in liver cancer. Experiments were performed using human Huh-7 and HepG2 cell lines, incubating cells with IL-8 and conducting
\end{abstract}

Correspondence to: Dr Jilu Shen, Department of Clinical Laboratory, The Fourth Affiliated Hospital of Anhui Medical University, 372 Tunxi Road, Hefei, Anhui 230000, P.R. China

E-mail: shenjilu@126.com

${ }^{*}$ Contributed equally

Key words: liver cancer, CXC chemokine receptor 1, CXC chemokine receptor 2 , interleukin- 8 , invasion, metastasis in vitro migration and invasion assays. The results showed that the wound healing activity and migration of Huh-7 and HepG2 cells were increased by IL-8. Pretreatment of the cells with anti-CXCR1 or anti-CXCR2 $(5 \mu \mathrm{M})$ for 30 min markedly inhibited IL-8-directed cell migration. Taken together, these results indicated that IL-8 promotes liver cancer cell migration via CXCR 1 and CXCR2 and that targeting the CXCR1/2 may be a potential strategy for liver cancer treatment.

\section{Introduction}

Liver cancer (LC) is the fifth most common type of cancer and the third leading cause of cancer-associated mortality worldwide (1). The clinical outcomes of patients with liver cancer remain poor, which is largely due to the high frequency of tumor recurrence and distant metastasis following surgical resection. Therefore, a better understanding of the molecular mechanisms of liver cancer is necessary to improve the prognosis of this disease (2).

Several studies have shown that mutual interactions between the tumor and its microenvironment contribute to tumor progression (3); uncontrolled inflammation also serves an important role in promoting malignant progression and metastasis (4). Chemokines, as an important class of non-resolving inflammatory factors, are important in tumor progression and metastasis and represent a potential tool for tumor detection $(5,6)$. Increasing experimental evidence has shown that chemokines produced in the tumor microenvironment serve critical roles in cancer-related inflammation, and promote invasion and metastasis in human cancer (7-9). In particular, IL-8 is a multifunctional CXC chemokine that affects human neutrophil functions including chemotaxis, enzyme release and the expression of surface adhesion molecules, and promotes tumor angiogenesis and metastasis through binding to its receptors CXCR1 and CXCR2 (10).

CXCR1 and CXCR2, as two important members of the CXC chemokine receptor family, were successfully cloned by Holmes et al (11) and Murphy and Tiffany (12), respectively. They are located on chromosome 2q35 and have a homology of $\leq 77 \%$. CXCR1 and CXCR2 combine with their common ligand, IL-8, with a high affinity and then induce leukocyte chemotaxis, calcium ion flow, cell proliferation and migration, and the regulation of angiogenesis (13). Numerous studies have 
suggested that CXCR $1 / 2$ is overexpressed in a wide variety of cancer types $(14,15)$, and serves a critical role in the pathogenesis, growth, invasion, metastasis, angiogenesis and drug resistance of malignant melanoma (16), breast cancer (17), prostate cancer (18), pancreatic cancer, colon cancer (19), gastric carcinoma (20) and epithelial ovarian cancer (21).

Previous studies have shown that IL- 8 and its receptors have an important regulatory role in the occurrence, proliferation, invasion and metastasis of cancer, and are involved in the regulation of tumor stem cell self-renewal. However, little is known about the expression and functional roles of IL-8, CXCR1 and CXCR2 in the progression of liver cancer, or the downstream signaling pathways that mediate IL-8-directed migration in liver cancer. In order to investigate the expression and functional roles of IL-8, CXCR1 and CXCR2 in the proliferation and invasion of liver cancer, the present study aimed to investigate the effect of their expression and define their roles in vitro.

\section{Materials and methods}

Human tissues. A total of 46 patients with liver cancer, who underwent surgery conducted by the same surgical team in the Department of the Fourth Affiliated Hospital of Anhui Medical University (Hefei, China) between October 2014 and September 2015 and agreed to participate in the study, were recruited. There were 25 men and 20 women, with an age range of 37-71 years old. Each subject had undergone a percutaneous liver biopsy at the Fourth Affiliated Hospital of Anhui Medical University. The standards for diagnosis of liver cancer have been previously described in The Standard for Diagnosis and Treatment of Primary Hepatocellular Carcinoma (2011 edition) (22). A total of 30 patients with liver cirrhosis, including 17 men and 13 women, with an age range of 33-71 years old, were considered for analysis. All cases were diagnosed using pathological, CT, ultrasound and clinical data. Another 28 blood donors from the City Center Blood Station with normal physical examination results, were selected as normal controls, which comprised 16 men and 12 women, aged between 28 and 69 years old.

Cell lines. The Huh-7 and HepG2 human liver cancer cell lines were purchased from the Shanghai Institute of Biological Sciences, Chinese Academy of Sciences. The HepG2 cell line used in the present study has been authenticated by STR profiling. The human Huh-7 and HepG2 cell lines were cultured in DMEM (Hyclone; GEHealthcare Life Sciences) supplemented with $10 \%$ fetal bovine serum (FBS; Gibco; Thermo Fisher Scientific, Inc.) at $37^{\circ} \mathrm{C}$ with $5 \% \mathrm{CO}_{2}$. In order to observe the effect of IL- 8 on the migration and invasion of liver cancer cell lines, the Huh-7 and HepG2 cells $\left(1.0 \times 10^{6}\right)$ were incubated with IL-8 at $37^{\circ} \mathrm{C}$ for 24 and $48 \mathrm{~h}$. In certain cases, the Huh-7 and HepG 2 cells were pretreated with $5 \mu \mathrm{M}$ anti-CXCR1 (1:400, cat. no. ab137351; Abcam) or anti-CXCR2(1:400; cat. no. ab14935; Abcam) for $30 \mathrm{~min}$ at $37^{\circ} \mathrm{C}$ to inhibit the function of CXCR1 or CXCR2, and the migration and invasion were measured in vitro via a wound healing assay and Transwell assay.

Wound healing assay. For the wound healing migration assay, $5 \times 10^{5}$ cells/well were seeded in 6-well plates and incubated until
90\% confluence. The cell monolayer was scratched with a fine pipette tip, and then cultured in serum-free medium containing IL-8 for a further 24 and $48 \mathrm{~h}$. Images were captured along the scrape line using a microscope (cat. no. CKX31; Olympus Corporation). The results are expressed as the relative scratch width, based on the distance migrated relative to the original scratch width. The experiment was performed in triplicate.

Migration and invasion assays. The migration and invasion assays were performed using a QCM 24-Well Cell Invasion Assay kit with 8- $\mu \mathrm{m}$ membranes (EMD Millipore). The cells ( $1 \times 10^{5}$ cells/well) in $150 \mu 1$ serum-free medium were seeded into the upper chamber. The same medium containing IL- 8 was used as a chemoattractant in the lower chamber. Following culture for $48 \mathrm{~h}$ at $37^{\circ} \mathrm{C}$, the upper surface of the Transwell membrane was wiped gently with a cotton swab to remove non-migrating cells. Those cells that had invaded to the lower surface of the membrane were fixed in methanol and stained with $0.1 \%$ crystal violet solution, followed by image capture and counting under an inverted microscope.

Detection of the mRNA expression levels of CXCR1, CXCR2 and $I L-8$ by reverse transcription $(R T)$-semi-quantitative $P C R$. The hepatic mRNA expression levels of CXCR1, CXCR2 and CXCL8 were assessed by RT-qPCR analysis. The details were as follows: Total RNA of the tissue samples was extracted from cells using TRIzol (Invitrogen; Thermo Fisher Scientific, Inc.) according to the manufacturer's protocol. The reverse transcription reaction was performed using the RevertAid First Strand cDNA Synthesis kit. Equal quantities of cDNA were submitted to PCR in the presence of SYBR Green Real-time PCR Master mix (Toboyo Life Science) and run in a real-time PCR detection machine with ABI 7500 PRISM (Applied Biosystems, Sunnyvale, CA, USA). The PCR conditions were as follows: Initial denaturation at $94^{\circ} \mathrm{C}$ for $5 \mathrm{~min}$, followed by 30 cycles of $94^{\circ} \mathrm{C}$ for $30 \mathrm{sec}$, annealing at a temperature in accordance with the primer sequence for $30 \mathrm{sec}$, and then $72^{\circ} \mathrm{C}$ for $30 \mathrm{sec}$, with a final extension at $72^{\circ} \mathrm{C}$ for $10 \mathrm{~min}$. The PCR products were analyzed using $2 \%$ agarose gel electrophoresis, stained with ethidium bromide and visualized under UV illumination. Realtime qPCR analysis was performed with specific primers for CXCR1, CXCR2 and CXCL8 (Table I). The housekeeping gene GAPDH was used as an internal control. Densitometry analysis was performed using Quantity One software (version 4.62; Bio-Rad Laboratories, Inc.).

Western blot analysis. In brief, liver cancer cells were lysed using radioimmunoprecipitation assay buffer (BIOSS) for $30 \mathrm{~min}$ on ice. The cell lysate was centrifuged at $12,000 \mathrm{x} \mathrm{g}$ for $20 \mathrm{~min}$ at $4^{\circ} \mathrm{C}$ and the supernatant was collected. Protein concentration was determined by $\mathrm{BCA}$ protein assay. A total of $30 \mu \mathrm{g}$ protein/lane was separated by SDS-PAGE on a $10 \%$ gel and transferred onto nitrocellulose filter membranes. Following blocking with 5\% skim milk for $1 \mathrm{~h}$ at room temperature, the membranes were incubated with primary antibodies overnight at $4^{\circ} \mathrm{C}$, and then with secondary antibodies for $2 \mathrm{~h}$ at room temperature. The primary antibodies used were as follows: Rabbit polyclonal anti-CXCR1 
Table I. Primer sequences of CXCR1, CXCR2 and GAPDH.

\begin{tabular}{lllc}
\hline Gene & \multicolumn{1}{c}{ Sense $(5 \rightarrow 3)$} & \multicolumn{1}{c}{ Antisense $(5 \rightarrow 3)$} & Length $(\mathrm{bp})$ \\
\hline CXCR 1 & CAGATCCACAGATGTGGGAT & AGCAGCCAAGACAAACAAACTT & 468 \\
CXCR2 & CTTTCTACTAGATGCCGC & AGATGCTGAGACATATGAATTT & 417 \\
IL-8 & CTTTGTCCATTCCCACTTCTGA & TCCCTAACGGTTGCCTTTGTAT & 306 \\
GAPDH & ACCACAGTCCATGCCATCAC & TCCACCACCCTGTTGCTGTA & 452
\end{tabular}

CXC chemokine receptor; IL-8, interleukin-8.

(1:2,000; cat. no. ab137351; Abcam) and anti-CXCR2 (1:2,000; cat. no. ab14935; Abcam) antibodies; monoclonal mouse anti-GAPDH (1:2,000; cat. no. TA-08; Zhongshan Jinqiao Biotechnology) and $\beta$-actin (1:2,000, cat. no. TA-09; Zhongshan Jinqiao Biotechnology, Beijing, China). The goat anti-mouse (cat. no. ZB2305; 1:10,000) and anti-rabbit secondary antibodies (cat. no. ZB2301; 1:10,000) were purchased from Zhongshan Jinqiao Biotechnology. The protein bands were visualized by ECL reagents (cat. no. C05-07003; BIOSS). Images were captured and the intensity of the bands was quantitated with the Bio-Rad Versa Doc imaging system (Bio-Rad Laboratories, Inc.).

Statistical analysis. Data are shown as the mean \pm standard deviation. Student's t-test was used to compare two independent groups of data. Differences among the three groups were analyzed using one-way analysis of variance. Multiple comparisons between the groups were performed using the Bonferroni correction method. Correlation between continuous variables was determined using Pearson's correlation coefficient. Chi-square tests were applied to analyze the relationships among immunohistochemical staining of IL-8, CXCR1 and CXCR2. All statistical analyses were performed using SPSS software (version 16.0; SPSS, Inc.). $\mathrm{P}<0.05$ was considered to indicate a statistically significant difference.

\section{Results}

Association between the MRNA expression levels of CXCRI, CXCR2 and IL-8 and the clinicopathological characteristics of liver cancer. The clinical analysis revealed that the mRNA levels of CXCR1, CXCR2 and IL-8 had no significant correlation with age or sex in patients with liver cancer $(\mathrm{P}>0.05)$, but were associated with the depth of tumor infiltration, lymph node or distant metastasis and TNM stage $(\mathrm{P}<0.05)$. The mRNA expression levels of CXCR1, CXCR2 and IL-8 were significantly increased in patients with liver cancer with deep invasion, lymph node or distant metastasis and a late TNM stage $(\mathrm{P}<0.05$; Table II).

mRNA expression levels of CXCR1, CXCR2 and IL-8 are increased in the peripheral blood mononuclear cells (PBMCs) of patients with liver cancer. The mRNA expression levels of CXCR1, CXCR2 and IL- 8 were detected in each patient with liver cancer. As shown in Fig. 1A-C, the mRNA levels of CXCR1, CXCR2 and IL- 8 were significantly increased in the PBMCs of patients with liver cancer compared with those of the controls $(\mathrm{P}<0.01)$. Additionally, it was found that the mRNA levels of CXCR1 and IL- 8 increased with the progress of liver disease. The mRNA levels of CXCR1 and IL-8 were significantly increased in patients with cirrhosis compared with those in normal controls $(\mathrm{P}<0.05)$. The mRNA levels of CXCR1 and IL-8 were higher in patients with liver cancer than in those with cirrhosis. The mRNA levels of CXCR2 did not differ significantly between the two groups.

Protein expression of CXCR1, CXCR2 and IL-8 is augmented in liver cancer. The immunohistochemical staining (Fig. 2) showed that the CXCR1 (Fig. 2B), CXCR2 (Fig. 2E) and IL-8 (Fig. 2H) proteins were apparent in almost all hepatocytes, infiltrating inflammatory cells, vascular endothelial cells and bile duct cells, and CXCR1, CXCR2 and IL- 8 were localized in the cytoplasm. In addition, the intensity of CXCR1, CXCR2 and IL-8 staining in liver cancer tissues was higher than that in the controls. Image analysis of 30 liver cancer samples revealed that the positive rate of CXCR1 expression was $86.7 \%$ (26/30), in which the proportions of weak and strong expression accounted for $30 \%(9 / 30)$ and $56.7 \%$ (17/30), respectively. The positive rate of CXCR2 expression was $76.7 \%$ (23/30), in which the proportion of weak and strong expression accounted for $26.7 \%(8 / 30)$ and $50 \%(15 / 30)$, respectively. The positive rate of IL-8 expression was $93.3 \%$ (28/30), in which the proportions of weak and strong expression accounted for $33.3 \%(10 / 30)$ and $60 \%(18 / 30)$, respectively. The positive rates of CXCR1, CXCR 2 and IL- 8 in 12 normal hepatic tissues were $33.3 \%(4 / 12), 25 \%(3 / 12)$ and $41.7 \%(5 / 12)$, respectively. The semi-quantitative analysis revealed that the relative mean densities of hepatic CXCR1 (Fig. 2C), CXCR2 (Fig. 2F) and IL-8 (Fig. 2I) staining in liver cancer were significantly increased compared with those in normal liver tissues $(\mathrm{P}<0.05$; Fig. 2A-I).

Expression of IL-8 is positively associated with the expression of CXCR1 and CXCR2 in liver cancer. The clinical findings in the present study suggested that the mRNA levels of IL-8, CXCR1 and CXCR2 were increased in patients with liver cancer, therefore the relationship between the expression of IL-8 and the expression of CXCR1 and CXCR2 was further examined. Statistical analysis revealed that the mRNA expression of IL- 8 was positively associated with that of CXCR1 $(r=0.618 ; \mathrm{P}<0.05)$ and CXCR2 
Table II. Association between the mRNA expression of CXCR1, CXCR2 and IL-8 and the clinicopathological parameters of patients with liver cancer.

\begin{tabular}{lllll}
\hline Variable & $\mathrm{n}$ & CXCR1 mRNA (fold change) & CXCR2 mRNA (fold change) & IL8 mRNA (fold change)
\end{tabular}

$\begin{array}{lcc}\text { Age (years) } & & \\ \leq 50 & 19 & 0.9874 \pm 0.2197 \\ >50 & 27 & 0.9389 \pm 0.1815 \\ \text { P-value } & & 0.418\end{array}$

Gender

$\begin{array}{lcc}\text { Male } & 26 & 0.9781 \pm 0.2081 \\ \text { Female } & 20 & 0.9340 \pm 0.1845 \\ \text { P-value } & & 0.459\end{array}$

Depth of infiltration

$\begin{array}{lcc}\text { T1-T2 } & 19 & 0.8721 \pm 0.1480 \\ \text { T3-T4 } & 27 & 1.0200 \pm 0.2069 \\ \text { P-value } & & 0.011\end{array}$

Lymph node metastasis

$\begin{array}{lcc}\text { No } & 21 & 0.8695 \pm 0.1559 \\ \text { Yes } & 25 & 1.0340 \pm 0.1997 \\ \text { P-value } & & 0.004\end{array}$

Distant metastasis

$\begin{array}{lcccc}\text { No } & 36 & 0.9056 \pm 0.1507 & 0.8528 \pm 0.1383 & 1.7004 \pm 0.1736 \\ \text { Yes } & 10 & 1.1510 \pm 0.2322 & 1.0560 \pm 0.1585 & 2.1645 \pm 0.2638 \\ \text { P-value } & & 0.009 & <0.001 & <0.001 \\ \text { TNM stage } & & & \\ \text { I+II } & 16 & 0.8525 \pm 0.1335 & 0.7987 \pm 0.1119 & 1.6064 \pm 0.1580 \\ \text { III+IV } & 30 & 1.0157 \pm 0.20394 & 0.9493 \pm 0.1655 & 1.9053 \pm 0.2665 \\ \text { P-value } & & 0.006 & 0.002 & <0.001\end{array}$

All data are presented as the mean $\pm \mathrm{SD}$ and were analyzed with the two-tailed unpaired t-test. CXCR, CXC chemokine receptor; IL-8, interleukin-8.
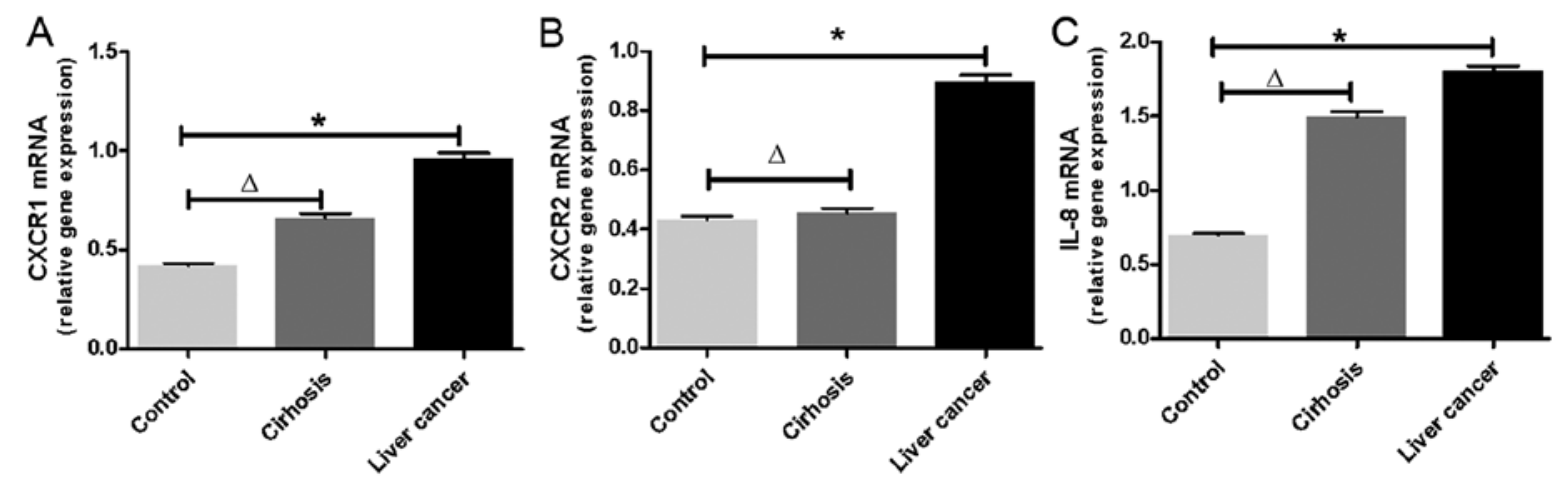

Figure 1. mRNA expression of CXCR1, CXCR2 and IL-8 in the PBMCs of patients with liver cancer.(A) mRNA levels of CXCR1 were detected by RT-qPCR analysis in the PBMCs of patients with liver cancer, patients with cirrhosis and controls. (B) mRNA levels of CXCR2 were detected by RT-qPCR analysis in the PBMCs of patients with liver cancer, patients with cirrhosis and controls. (C) mRNA levels of IL-8 were detected by RT-qPCR analysis in the PBMCs of patients with liver cancer patients, patients with cirrhosis and controls; ${ }^{P} \mathrm{P}<0.01 ;{ }^{\wedge} \mathrm{P}<0.05$. All data are presented as the mean $\pm \mathrm{SD}$ and were analyzed with one-way ANOVA. CXCR, CXC chemokine receptor; IL-8, interleukin-8; PBMCs, peripheral blood mononuclear cells; RT-qPCR, reverse transcription-quantitative polymerase chain reaction.

$(\mathrm{r}=0.569 ; \mathrm{P}<0.05)$. CXCR1 and CXCR2 mRNA gradually increased with the increased expression of IL-8 in liver cancer (Fig. 3).
IL-8 promotes human liver cancer cell invasion and metastasis through CXCRI/2 receptors. To determine whether IL-8 induces the expression of CXCR1 or CXCR2 in Huh-7 and 

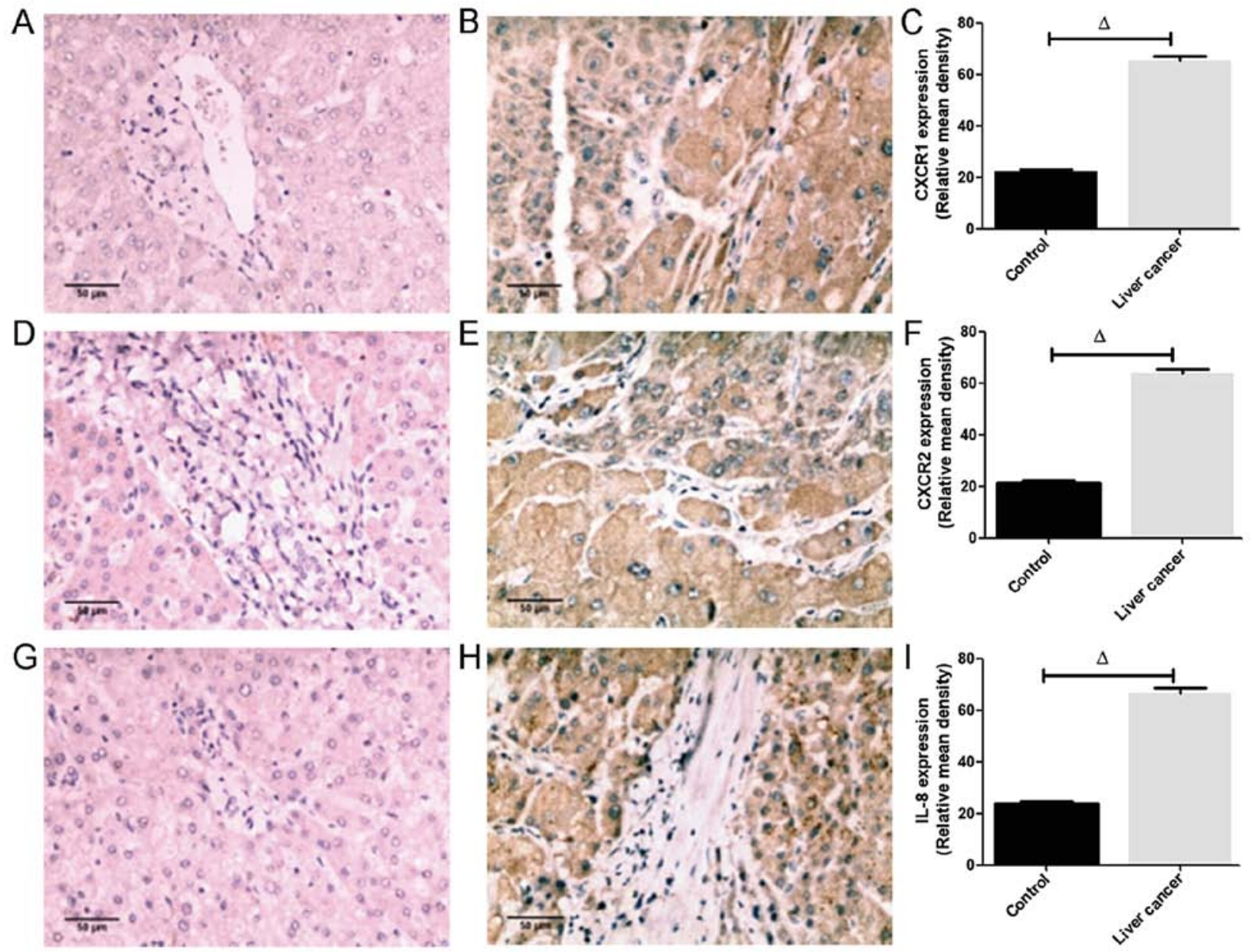

Figure 2. Protein expression of CXCR1, CXCR2 and IL-8 in liver biopsy specimens of liver cancer (magnification, x400). Protein expression of CXCR1 in (A) normal controls and (B) patients with liver cancer. (C) Relative mean density analysis showed differences in hepatic CXCR1 staining between liver cancer $(n=30)$ and control tissues $(n=12)$. Protein expression of CXCR2 in (D) normal controlsand (E) patients with liver cancer. (F) Relative mean density analysis showed the difference in hepatic CXCR2 staining between liver cancer $(n=30)$ and controls $(n=12)$. Protein expression of IL-8 in (G) normal controls and $(\mathrm{H})$ patients with liver cancer. (I) Relative mean density analysis showed the difference in hepatic IL-8 staining between liver cancer ( $\mathrm{n}=30)$ and control tissues $(\mathrm{n}=12)$. All data are presented as the mean $\pm \mathrm{SD}$ and were analyzed with the two-tailed unpaired $\mathrm{t}$-test $\left({ }^{\triangle} \mathrm{P}<0.05\right)$. CXCR, CXC chemokine receptor; IL-8, interleukin-8.
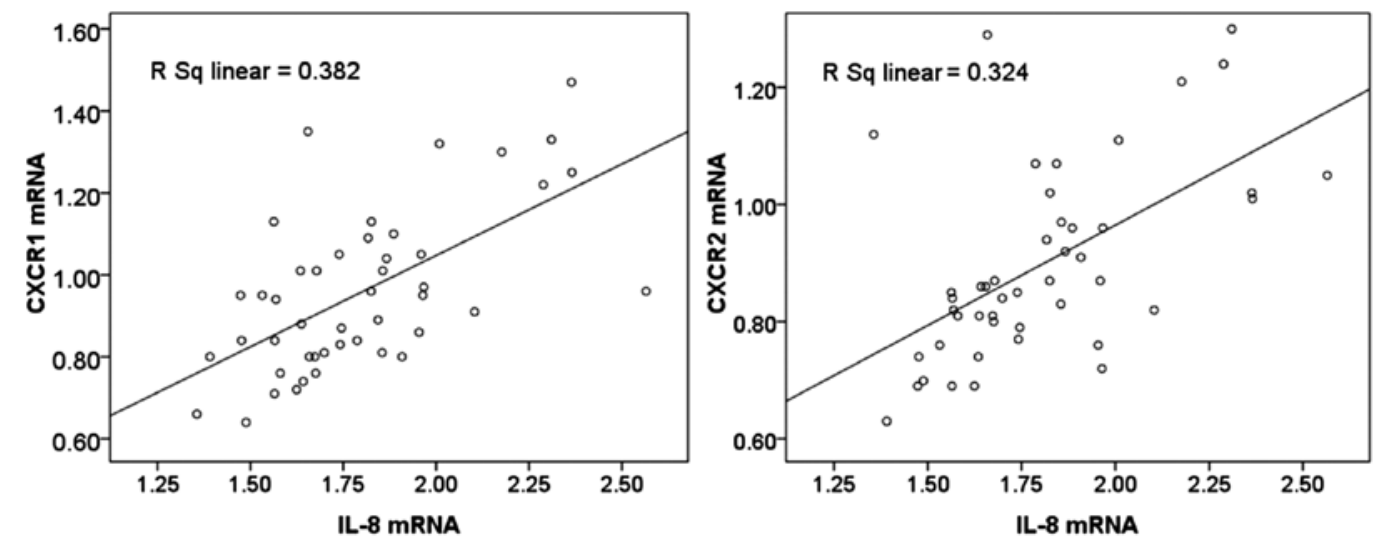

Figure 3. Hepatic expression of IL-8 and correlation with CXCR1 and CXCR2. The mRNA expression of IL-8 was positively associated with that of CXCR1 $(\mathrm{r}=0.618, \mathrm{P}<0.05)$ and CXCR2 $(\mathrm{r}=0.569 ; \mathrm{P}<0.05)$. CXCR, CXC chemokine receptor; IL-8, interleukin-8.

HepG2 cells, the cell lines were treated with IL-8 for $24 \mathrm{~h}$ and then subjected to RT-qPCR and western blot analyses (Fig. 4A). It was found that IL-8 significantly increased the mRNA expression of CXCR1 and CXCR2. It has been shown that IL-8 directs the migration and invasion of human liver cancer cells. In the present study, the effect of IL-8 on the motility of Huh-7 and HepG2 cells was verified. The wound-healing activity (Fig. 4B and C) and the migration and 

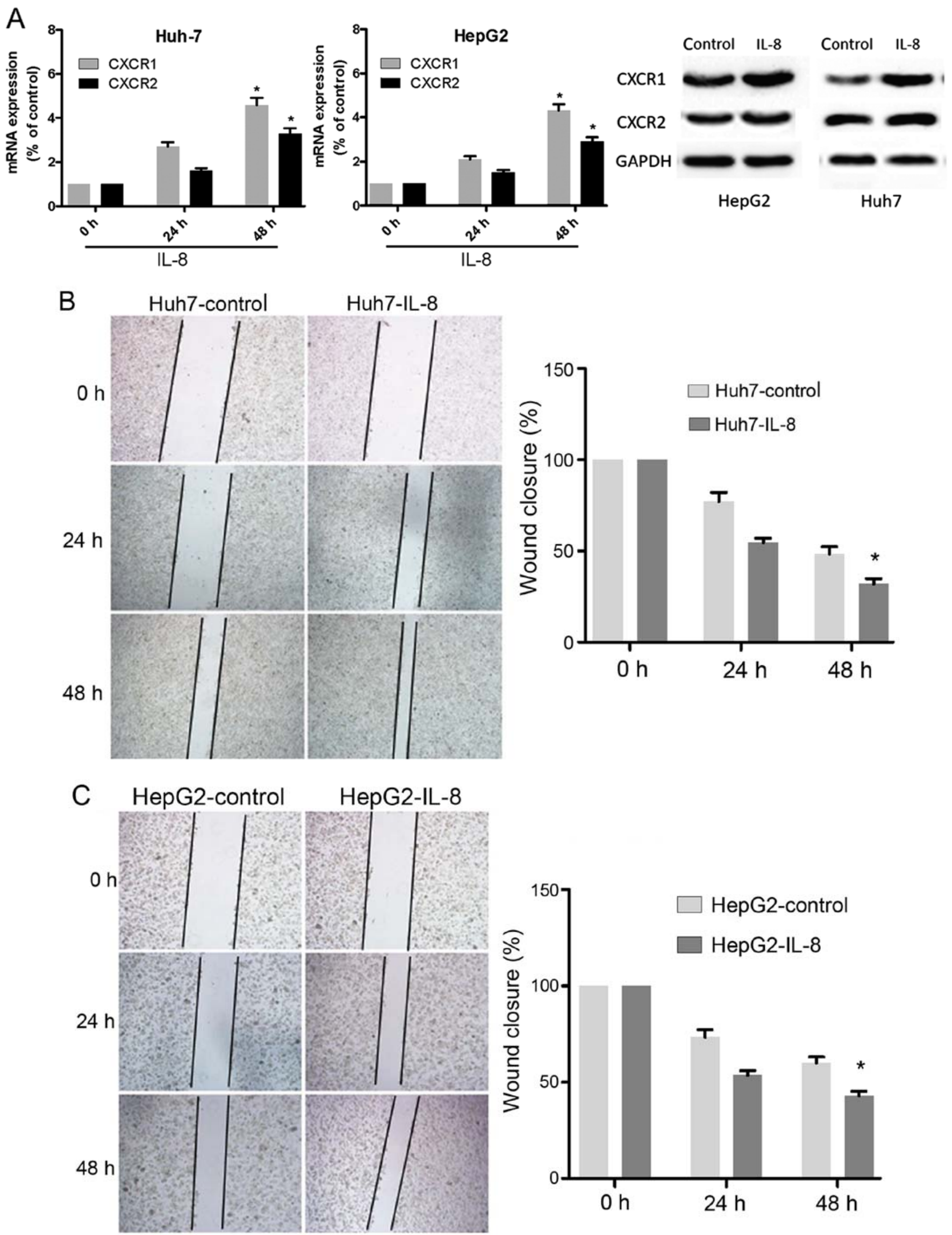

Figure 4. IL-8 promotes human liver cancer cell invasion and metastasis through CXCR1/2 receptors. (A) Huh7 and HepG2 cells were treated with IL-8 for $24 \mathrm{~h}$, and the expression levels of CXCR1 and CXCR2 were detected by reverse transcription-quantitative polymerase chain reaction analysis and western blotting. (B) Huh7 cell migration 24 and $48 \mathrm{~h}$ after IL-8 stimulation was assessed using wound healing assays. (C) HepG2 cells migration after 24 and $48 \mathrm{~h}$ after IL-8 stimulation was assessed using wound healing assays. Right panel shows quantitation of the wound closure shown in the left panel. The y-axis represents the percentage of wound closure at 24 or $48 \mathrm{~h}$ post-wound introduction, as determined using ImageJ software. 

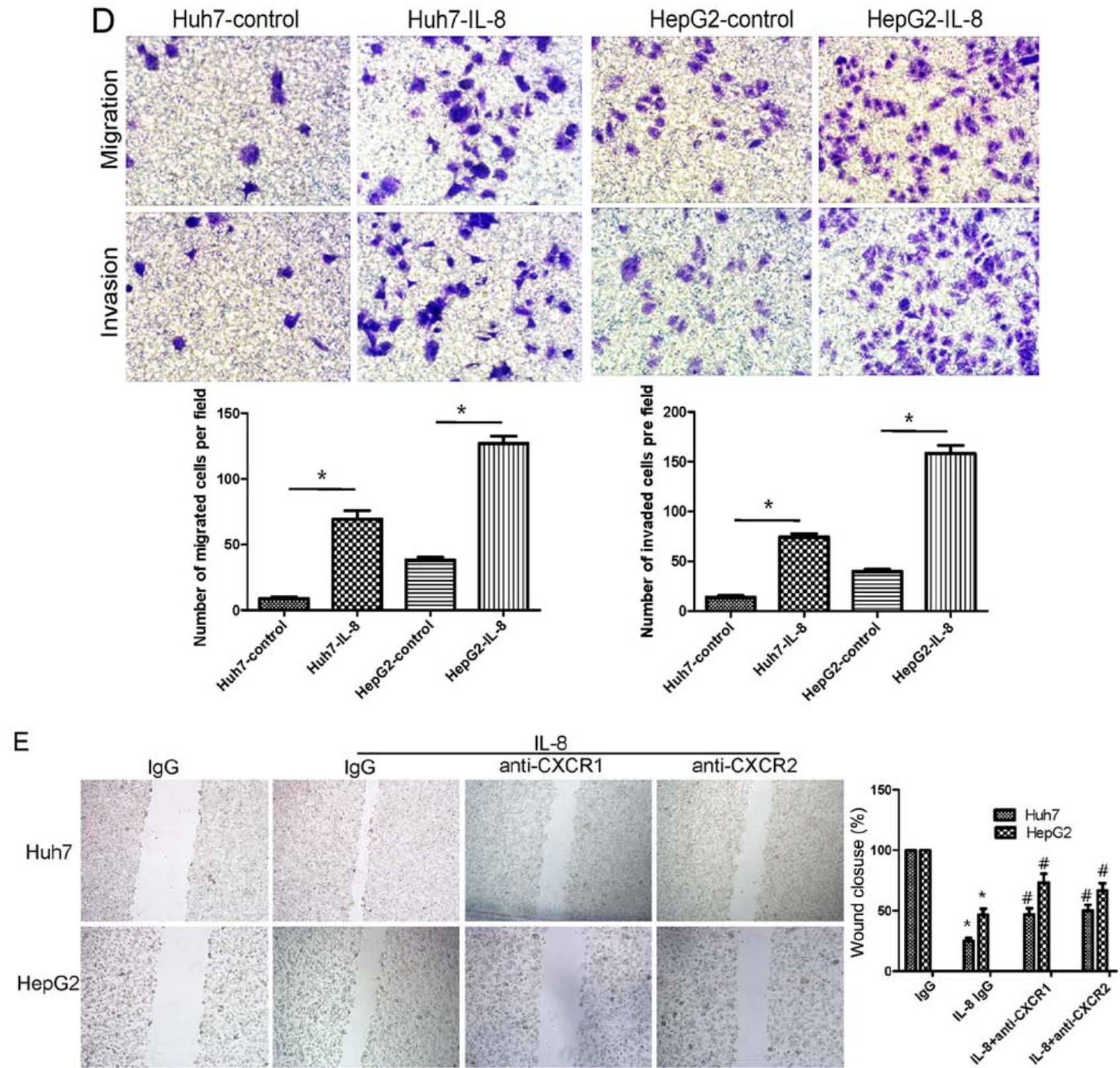

Figure 4. Continued. (D) Transwell assay analysis of the migration and invasion abilities of Huh7 and HepG2 cells (magnification, x100). (E) Huh7 and HepG2 cells were pretreated with CXCR1 or CXCR2 antibodies $(5 \mu \mathrm{M})$ for $30 \mathrm{~min}$ followed by stimulation with $10 \mathrm{ng} / \mathrm{ml} \mathrm{IL}-8$ for $24 \mathrm{~h}$, and in vitro migration was measured with the wound healing assay. Results are expressed as the mean $\pm \mathrm{SEM}$; ${ }^{*} \mathrm{P}<0.05$, compared with the control; ${ }^{*} \mathrm{P}<0.05$, compared with the IL-8-treated group. CXCR, CXC chemokine receptor; IL-8, interleukin-8.

invasion of Huh-7 and HepG2 cells (Fig. 4D) were increased by IL-8 after 24 and $48 \mathrm{~h}$. Pretreatment of the cells with anti-CXCR1 or anti-CXCR2 $(5 \mu \mathrm{M})$ for 30 min markedly inhibited IL-8-directed cell migration and invasion (Fig. 4E). This suggested that IL-8 promoted liver cancer cell migration via the CXCR1 and CXCR2 receptors.

\section{Discussion}

Treatment for many patients with early-stage liver cancer is successful, however, patients with distant metastasis have a poor prognosis with conventional therapy. When the disease is recurrent or metastatic, conventional chemotherapy or radiotherapy has limited benefit. Increasing epidemiologic and experimental evidence has shown that inflammation serves an important role in promoting the malignant progression and metastasis of liver cancer. Chemokines and their receptors produced in the tumor microenvironment serve critical roles in cancer-related inflammation and promote invasion and metastasis in human cancer.

CXCR 1 and CXCR2, as two important members of the CXC chemokine receptor family, have been shown to contribute to human tumor growth invasion and metastasis through binding their common ligand IL-8. Considerable data demonstrate that tumor cells express widely functional chemokine receptors to facilitate tumor progression. However, there is no direct in vivo evidence that CXCR1 and CXCR2 are expressed in liver cancer.

In the present study, the mRNA levels of CXCR1, CXCR2 and IL-8 in PBMCs from 46 patients with liver cancer were 
detected by RT-qPCR. It was found that the mRNA levels of CXCR1, CXCR2 and IL-8 were highest in patients with liver cancer and lowest in the normal control group $(\mathrm{P}<0.05)$. With the progression of liver cancer, the mRNA levels of CXCR1, CXCR2 and IL- 8 increased gradually, suggesting that these expression changes were significantly associated with advanced liver cancer progression. Correlation analysis was performed between IL- 8 and CXCR $1 / 2$ in liver cancer. The results revealed that the mRNA expression of IL- 8 was positively associated with that of CXCR1 $(r=0.618 ; \mathrm{P}<0.05)$ and CXCR2 ( $r=0.569 ; \mathrm{P}<0.05)$. The mRNA expression levels of CXCR1 and CXCR2 gradually increased as the expression of IL-8 was elevated in liver cancer. In addition, clinical analysis revealed that the mRNA expression levels of CXCR1, CXCR2 and IL-8 did not significantly correlate with the age or sex of patients with liver cancer $(\mathrm{P}>0.05)$, but were associated with the depth of tumor infiltration, lymph node or distant metastasis and TNM stage $(\mathrm{P}<0.05)$. The mRNA expression levels of CXCR1, CXCR2 and IL-8 were significantly increased in patients with liver cancer with T3-T4, lymph node metastasis, distant metastasis and advanced tumor stage (pTNM stages III-IV), suggesting that CXCR1, CXCR2 and IL-8 were associated with the clinical stage, lymph node metastasis and distant metastasis of liver cancer. These findings were concordant with those of Ren et al (23), who detected the serum levels of IL-8 in 59 patients with liver cancer and 15 healthy subjects using ELISAs. The results showed that the serum level of IL-8 was significantly elevated in patients with liver cancer and associated with larger tumor size $(>5 \mathrm{~cm})$, absence of a tumor capsule, presence of venous invasion and advanced pathological tumor-node-metastasis stage. Akiba et al (24) provided evidence that HCC cells are a major producer of IL-8 in tissues, and cases with a high level of IL-8 in cancerous tissues had a significantly higher frequency of portal vein invasion, venous invasion and bile duct invasion. This suggests that IL- 8 may serve an important role in the invasion and metastasis of liver cancer.

The present study also detected the protein expression of CXCR1, CXCR2 and IL-8 using immunohistochemical methods. The results showed that the CXCR1, CXCR2 and IL-8 proteins were mainly expressed in the cytoplasm of hepatoma cells, with higher intensities than in the normal controls $(\mathrm{P}<0.01)$; this was in accordance with the data presented by Wang et al (25). The present study found that the intensity of CXCR1, CXCR2 and IL-8 expressed was statistically significant between liver cancer and normal controls. These results further confirmed that the high level of IL-8 secreted by hepatoma cells induced high expression of CXCR1 and CXCR2 on hepatoma and inflammatory cells, which, in turn, is involved in the development, invasion and metastasis of liver cancer.

In order to further verify the hypothesis, experiments were performed using Huh-7 and HepG2 cell lines. The cell lines were incubated with IL-8 for $24 \mathrm{~h}$, and the expression of CXCR1 and CXCR2 was detected by RT-qPCR and western blot analyses. It was found that IL-8 significantly increased the expression of CXCR1 and CXCR2 in Huh-7 and HepG2 cell lines. To determine whether IL- 8 promotes liver cancer migration and invasion via CXCR1 and CXCR2, the Huh-7 and HepG2 cells were incubated with IL-8 for 24 and 48 h, following which the wound-healing activity and the migration and invasion of Huh-7 and HepG2 cells were increased. Pretreatment of cells with anti-CXCR1 or anti-CXCR2 $(5 \mu \mathrm{M})$ for 30 min markedly inhibited the IL-8-directed migration of Huh-7 and HepG2 cells. Xue et al (26) analyzed the expression profiles of 18 chemokine receptors on four HCC cell lines of lower to higher metastatic potentials of (SMMC-7721, MHCC97-L, MHCC97-H and HCCLM6) using RT-PCR analysis and found that chemokine receptors are closely associated with the metastatic potential of HCC. Huang et al (27) demonstrated that the incubation of HCC cells with IL-8 led to the increased expression of FOXC1 via activation of phosphoinositide 3-kinase (PI3K) signaling to AKT and hypoxia-inducible factor $1 \alpha$. Increased expression of FOXC1 can lead to the transactivation of CXCR1 and CCL2, promoting inflammation and the invasive and metastatic abilities of HCC cells. Therefore, a high level of IL-8 (CXCL8) may regulate local and systemic inflammatory responses in patients with liver cancer through the upregulation of CXCR1/2, and be involved in the development of liver cancer through activation of the PI3K/Akt/HIF-1 $\alpha$ signaling pathway (16). However, the exact mechanism underlying the involvement of CXCR1, CXCR2 and IL-8 in liver cancer remains to be elucidated and requires further investigation.

In conclusion, the results of the present study demonstrate that the mRNA and protein levels of CXCR1, CXCR2 and IL-8 in patients with liver cancer were increased, and were significantly associated with the clinical stage, lymph node metastasis and distant metastasis. Correlation analysis indicated that the expression of IL-8was positively associated with the expression of CXCR1 and CXCR2 in liver cancer. In vitro, IL-8 was shown to induce Huh-7 and HepG2 cell migration and invasion via the increased expression of CXCR1 and CXCR2. Based on these findings, and other relevant reports, IL-8, CXCR1 and CXCR2 may be involved in the invasion and metastasis of liver cancer and may be useful in identifying patients with more aggressive tumors for neoadjuvant or adjuvant therapy.

\section{Acknowledgements}

Not applicable.

\section{Funding}

The current study was supported by Anhui Medical University (grant no. 2017zhyx14) and the Fund of the Fourth Affiliated Hospital of Anhui Medical University (grant no. 2015xkj049).

\section{Availability of data and materials}

The analyzed data sets generated during the study are available from the corresponding author on reasonable request.

\section{Authors' contributions}

JS designed the study. HB and YZ performed the experiments, SW, WF, WH, LY, YX, ZC and MY analyzed the data. HB, $\mathrm{YZ}$ and JS wrote the manuscript. All authors reviewed the manuscript. 


\section{Ethics approval and consent to participate}

The present study was performed in strict accordance with the Ethics Committee of The Fourth Affiliated Hospital of Anhui Medical University of Anhui province and written informed consent was provided by each patient. All methods were performed in accordance with relevant guidelines and regulations.

\section{Patient consent for publication}

Not applicable.

\section{Competing interests}

The authors declare that they have no competing interests.

\section{References}

1. Yu SJ: A concise review of updated guidelines regarding the management of hepatocellular carcinoma around the world: 2010-2016. Clin Mol Hepatol 22: 7-17, 2016.

2. Omata M, Cheng AL, Kokudo N, Kudo M, Lee JM, Jia J, Tateishi R, Han KH, Chawla YK, Shiina S, et al: Asia-Pacific clinical practice guidelines on the management of hepatocellular carcinoma: A 2017 update. Hepatol Int 11: 317-370, 2017.

3. McAllister SS and Weinberg RA: The tumour-induced systemic environment as a critical regulator of cancer progression and metastasis. Nat Cell Biol 16: 717-727, 2014.

4. Zhang $\mathrm{H}$ and Xu X: Mutation-promoting molecular networks of uncontrolled inflammation. Tumour Biol 39: 1010428317701310, 2017.

5. Ruffini PA, Morandi P, Cabioglu N, Altundag K and Cristofanilli M: Manipulating the chemokine-chemokine receptor network to treat cancer. Cancer 109: 2392-2404, 2007.

6 . Karin N: Chemokines and cancer: New immune checkpoints for cancer therapy. Curr Opin Immunol 51: 140-145, 2018.

7. Dayer R, Babashah S, Jamshidi S and Sadeghizadeh M: Upregulation of CXC chemokine receptor 4-CXC chemokine ligand 12 axis ininvasive breast carcinoma: A potent biomarker predicting lymph node metastasis. J Cancer Res Ther 14: 345-350, 2018.

8. Mao TL, Fan KF and Liu CL: Targeting the CXCR4/CXCL12 axis in treating epithelial ovarian cancer. Gene Ther 24: 621-629, 2017.

9. Bronger H, Karge A, Dreyer T, Zech D, Kraeft S, Avril S, Kiechle $M$ and Schmitt M: Induction of cathepsin B by the CXCR3 chemokines CXCL9 and CXCL10 in human breast cancer cells. Oncol Lett 13: 4224-4230, 2017.

10. Hosono M, Koma YI, Takase N, Urakawa N, Higashino N, Suemune K, Kodaira H, Nishio M, Shigeoka M, Kakeji Y and Yokozaki H: CXCL8 derived from tumor-associated macrophages and esophageal squamous cell carcinomas contributes to tumor progression by promoting migration and invasion of cancer cells. Oncotarget 8: 106071-88, 2017.

11. Holmes WE, Lee J, Kuang WJ, Rice GC and Wood WI: Structure and functional expression of a human interleukin-8 receptor. Science 253: 1278-1280, 1991.
12. Murphy PM and Tiffany HL: Cloning of complementary DNA encoding a functional human interleukin- 8 receptor. Science 253: 1280-1283, 1991.

13. Bi HJ, Wang J, Huang HS and Liu JH: Influence of IFN on expression of chemokine receptor CXCR1, CXCR2 and their ligand IL-8 in the patients with chronic hepatitis B. Xi Bao Yu Fen Zi Mian Yi Xue Za Zhi 28: 422-425, 2012 (In Chinese).

14. Ha H, Debnath B and Neamati N: Role of the CXCL8-CXCR1/2 axis in cancer and inflammatory diseases. Theranostics 7: 1543-1588, 2017.

15. Liu Q, Li A, Tian Y, Wu JD, Liu Y, Li T, Chen Y, Han X and Wu K: The CXCL8-CXCR1/2 pathways in cancer. Cytokine Growth Factor Rev 31: 61-71, 2016.

16. Singh S, Sadanandam A, Varney ML, Nannuru KC and Singh RK: Small interfering RNA-mediated CXCR1 or CXCR2 knock-down inhibits melanoma tumor growth and invasion. Int J Cancer 126: 328-336, 2010.

17. Zhu H, Gu Y, Xue Y, Yuan M, Cao X and Liu Q: CXCR2+ MDSCs promote breast cancer progression by inducing EMT and activated T cell exhaustion. Oncotarget 8: 114554-114567, 2017.

18. Franz JM, Portela P, Salim PH, Berger M, Fernando Jobim L, Roesler R, Jobim M and Schwartsmann G: CXCR2 +1208 CT genotype may predict earlier clinical stage at diagnosis in patients with prostate cancer. Cytokine 97: 193-200, 2017.

19. Li A, Varney ML and Singh RK: Expression of interleukin 8 and its receptors in human colon carcinoma cells with different metastatic potentials. Clin Cancer Res 7: 3298-3304, 2001.

20. Li Z, Wang Y, Dong S, Ge C, Xiao Y, Li R, Ma X, Xue Y, Zhang Q, Lv J, et al: Association of CXCR1 and 2 expressions with gastric cancer metastasis in ex vivo and tumor cell invasion in vitro. Cytokine 69: 6-13, 2014.

21. Yung MM, Tang HW, Cai PC, Leung TH, Ngu SF, Chan KK, Xu D, Yang H, Ngan HY and Chan DW: GRO- $\alpha$ and IL-8 enhance ovarian cancer metastatic potential via the CXCR2-mediated TAK1/NFKB signaling cascade. Theranostics 8: 1270-1285, 2018.

22. Ministry of Health of the People's Republic of China: Standard for diagnosis and treatment of primary hepatocellular carcinoma (2011 edition). Chin J Hepatol 20: 419-426, 2012 (In Chinese).

23. Ren Y, Poon RT, Tsui HT, Chen WH, Li Z, Lau C, Yu WC and Fan ST: Interleukin-8 serum levels in patients with hepatocellular carcinoma: Correlations with clinicopathological features and prognosis. Clin Cancer Res 9: 5996-6001, 2003.

24. Akiba J, Yano H, Ogasawara S, Higaki K and Kojiro M: Expression and function of interleukin-8 in human hepatocellular carcinoma. Int J Oncol 18: 257-264, 2001.

25. Wang J, Han Z and Zhou N: Expression of CXCL8 and its receptors (CXCR1 and CXCR2) in peripheral blood neutrophils of chronic hepatitis B. Chin J Immunol: 375-379,383, 2015.

26. Xue TC, Chen RX, Ye SL, Sun RX, Chen J and Tang ZY: Different expressions of chemokine receptors in human hepatocellular carcinoma cell lines with different metastatic potentials. Zhonghua Gan Zang Bing Za Zhi 15: 261-265, 2007 (In Chinese).

27. Huang W, Chen Z, Zhang L, Tian D, Wang D, Fan D, Wu K and Xia L: Interleukin-8 induces expression of FOXC1 to promote transactivation of CXCR1 and CCL2 in hepatocellular carcinoma cell lines and formation of metastases in mice. Gastroenterology 149: 1053-1067.e14, 2015.

This work is licensed under a Creative Commons Attribution-NonCommercial-NoDerivatives 4.0 International (CC BY-NC-ND 4.0) License. 